\title{
Transmission Comparison of Ultra-Long Raman Fibre Laser Based Amplification with First and Dual Order Raman Amplification using 10x118 Gbit/s DP-QPSK
}

\author{
Mingming Tan, Pawel Rosa, Ian. D. Phillips, M.F.C. Stephens, Stylianos Sygletos, Andrew Ellis and Paul \\ Harper \\ Aston Institute of Photonic Technologies, Aston University, Aston Triangle, Birmingham B4 7ET, UK \\ Tel: (0121) 204 3512,e-mail: tanm@aston.ac.uk
}

\begin{abstract}
Experimental investigations of 10x118 Gbit/s DP-QPSK WDM transmission using three types of distributed Raman amplification techniques are presented. Novel ultra-long Raman fibre laser based amplification with second order counter-propagated pumping is compared with conventional first order and dual order counterpumped Raman amplification. We demonstrate that URFL based amplification can extend the transmission reach up to a distance of $7520 \mathrm{~km}$ in comparison with $5010 \mathrm{~km}$ and $6180 \mathrm{~km}$ using first order and dual order Raman amplification respectively.
\end{abstract}

Keywords: coherent fibre optic communications, Raman amplification, Raman fibre laser.

\section{INTRODUCTION}

In fibre optic communications, dual-polarisation quadrature-phase-shift keying (DP-QPSK) modulation with coherent detection is widely deployed due to its good balance between robustness against optical signal to noise ratio degradation (OSNR) and spectral efficiency [1,2]. In order to maximise the transmission distance, it is necessary to maintain an acceptable OSNR through the system. Distributed Raman amplification (DRA) reduces signal attenuation in the transmission span leading to a higher OSNR which can allow longer reach between the repeaters or longer total distance in unrepeatered systems [3]-[7]. Ultra-long Raman fibre laser (URFL) based amplification with fibre Bragg gratings (FBGs) can further reduce the signal power variation during transmission and has proved advantageous in comparison with EDFA based systems [8]-[10].

In this paper, we experimentally investigate the performances of 10x118 Gbit/s DP-QPSK WDM transmissions with coherent detection in an $83.5 \mathrm{~km}$ SMF-28 recirculating loop using three types of DRA. These are first order counter-pumped, dual order (both first and second order) counter-pumped, and novel URFL based amplification with $2^{\text {nd }}$ order counter pumping. To the best of our knowledge, this is the first experimental transmission comparison between first order or dual order Raman amplification and URFL based amplification. In particular, we show an improved reach of $7520 \mathrm{~km}$ using URFL based amplification, compared with $5010 \mathrm{~km}$ and $6180 \mathrm{~km}$ using first order and dual order Raman amplification, respectively.

\section{EXPERIMENTAL SETUP}

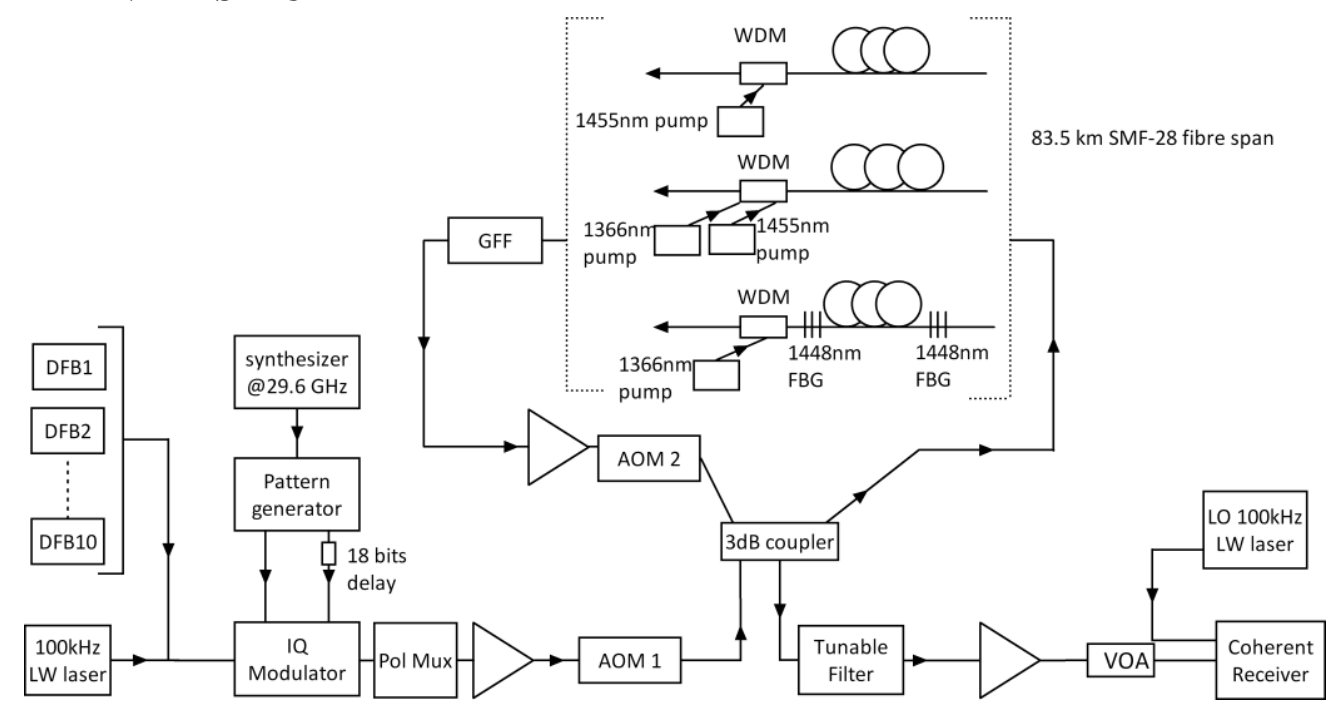

Figure 1. Experimental setup of DP-QPSK transmitter, recirculating loop and coherent receiver.

The experimental setup is shown in Figure 1. The transmitter consists of ten DFB lasers with $100 \mathrm{GHz}$ spacing ranging from $194.4 \mathrm{THz}(1542.142 \mathrm{~nm})$ to $193.5 \mathrm{THz}(1549.315 \mathrm{~nm})$. These were combined with a $100 \mathrm{kHz}$ linewidth, tunable laser through a polarization maintaining 50/50 coupler. The $100 \mathrm{kHz}$ linewidth, tunable laser was used as a "channel under test" while the corresponding DFB laser was switched off during the measurement 
of the channel. The combined signal was modulated with an IQ modulator driven by $29.6 \mathrm{Gbit} / \mathrm{s}, 2^{31}-1$ word length, normal and inverted PRBS patterns from the pattern generator. The clock rate of the system was chosen to give an integer number of bits delay between the normal and inverted PRBS pattern - in this case this was 18 bits. The resulting 29.6 Gbaud QPSK signals were polarisation division multiplexed (PDM) with a $\sim 10$ ns delay (equivalent to 296 bits) between two arms to create 10x118.4 Gb/s DP-QPSK signals and amplified by an EDFA before being launched into a recirculating loop.

The recirculating loop consisted of a single $83.5 \mathrm{~km}$ SMF-28 transmission span with a total loss of $17.6 \mathrm{~dB}$ (including $16.7 \mathrm{~dB}$ from SMF-28 fibre, $0.9 \mathrm{~dB}$ from $1 \times 3 \mathrm{WDM}$ in first order and dual order configurations, 0.7 $\mathrm{dB}$ from 1366/1550 nm WDMs and $0.2 \mathrm{~dB}$ from FBGs in URFL based configuration). To equalise the spectrum, a gain flattening filter (GFF) was included in the loop. The $\sim 11 \mathrm{~dB}$ loss from the GFF, AOM and $3 \mathrm{~dB}$ couplers was compensated using an EDFA at the end of the recirculation loop.

The three different types of Raman amplifications are shown schematically in Figure 1. For the first order Raman amplification, the span was counter-pumped by a $\sim 600 \mathrm{~mW}, 1455 \mathrm{~nm}$ source. This was used as a baseline comparison with the other DRA techniques discussed below.

In the dual order Raman amplification scheme, a $1 \times 3$ WDM coupler was used to combine counter-propagated $1366 \mathrm{~nm}$ and $1455 \mathrm{~nm}$ pumps. The pump powers at $1366 \mathrm{~nm}$ and $1455 \mathrm{~nm}$ into the fibre span (after WDM) were optimized to be $1016 \mathrm{~mW}$ and $10 \mathrm{~mW}$ respectively. This technique offers superior noise figure to standard first order only pumping due to higher $2^{\text {nd }}$ order and lower first order pump power which produces amplification further from the end of fibre [13,14].

To form a URFL based amplifier, a matched pair of high reflectivity ( $95 \%$ ) FBGs centered at $1448 \mathrm{~nm}$, with $3 \mathrm{~dB}$ bandwidths of $\sim 0.5 \mathrm{~nm}$ were used at the ends of the span. A counter-propagating highly de-polarised pump laser created an ultra-long fibre laser (by Raman amplification) at the wavelength specified by the FBGs. The resultant bi-directionally propagating $1448 \mathrm{~nm}$ laser together with the $1366 \mathrm{~nm}$ pump acted so as to amplify the C-band signals [10]-[12]. The $1366 \mathrm{~nm}$ pump power into the fibre span (after FBG) was optimized to $1091 \mathrm{~mW}$.

For the coherent receiver path, the WDM signal was demultiplexed using a tunable filter with $0.4 \mathrm{~nm}$ bandwidth. The resulting signal with output power of $+6 \mathrm{dBm}$ was combined with a $100 \mathrm{kHz}$ linewidth local oscillator (LO) in a polarisation diverse 90 degree optical hybrid [15]. Polarisation multiplexed I (in-phase) and $\mathrm{Q}$ (quadrature) signals were recovered using four high speed photodiodes and captured with a real-time oscilloscope with $80 \mathrm{GSample/s} \mathrm{sampling} \mathrm{rate} \mathrm{and} 36 \mathrm{GHz}$ bandwidth. The sampled traces were processed offline using digital signal processing (DSP) including channel correction and equalisation, electrical filtering, resampling, static chromatic dispersion compensation, clock recovery, constant modulus algorithm (CMA), frequency offset correction and phase recovery. $\mathrm{Q}^{2}$ factors were obtained from the error vector magnitude after averaging over 590k symbols.

\section{RESULTS AND DISCUSSIONS}

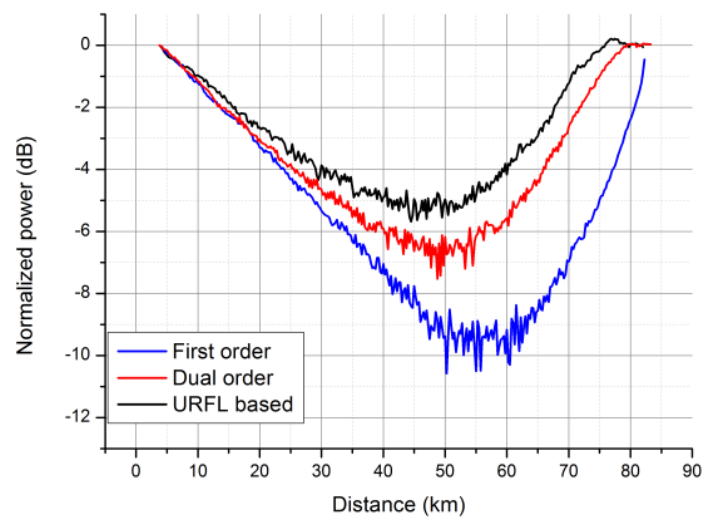

Figure 2. Signal power variations along the transmission span of three Raman amplification techniques.

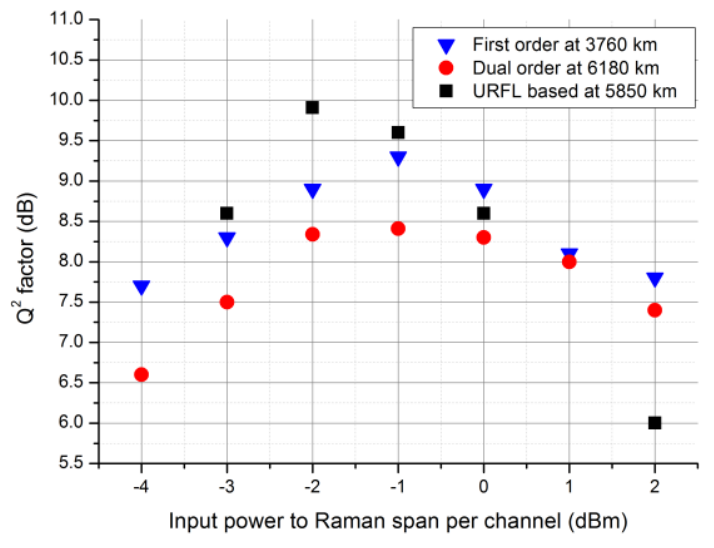

Figure 3. Input power sweep for different Raman amplifications techniques.

Signal power distributions along the transmission span (measured using a modified optical time-domain reflectometer (OTDR) [10]) for all Raman amplification techniques are shown in Figure 2. Peak-to-peak signal power variations using first order, dual order, and URFL based amplification were $\sim 9.5 \mathrm{~dB}, \sim 6.5 \mathrm{~dB}$ and $\sim 5 \mathrm{~dB}$, respectively. It is believed that the achievement of reduced signal power distribution using URFL based amplification is because unlike the other two techniques, the induced $1448 \mathrm{~nm}$ ultra-long fibre laser is bidirectionally propagating in a FBG cavity. This effect also reduces the noise figure of URFL based systems [12]. Figure 3 shows the input power per channel versus $Q^{2}$ factor using the three amplification techniques at the 
distances indicated. The optimum measured input power per channel for first order, dual order and the URFL based amplifications was similar and between -2 and $-1 \mathrm{dBm}$.

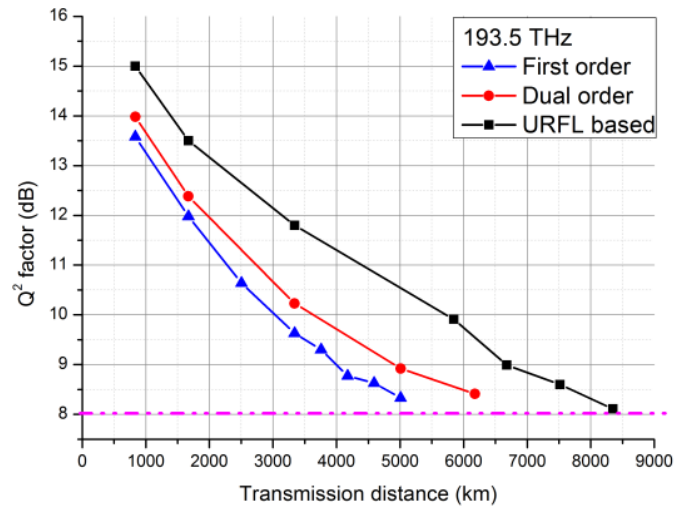

Figure 4. Transmission distances versus $Q^{2}$ factors for the $193.5 \mathrm{THz}$ channel using different Raman amplifications techniques.

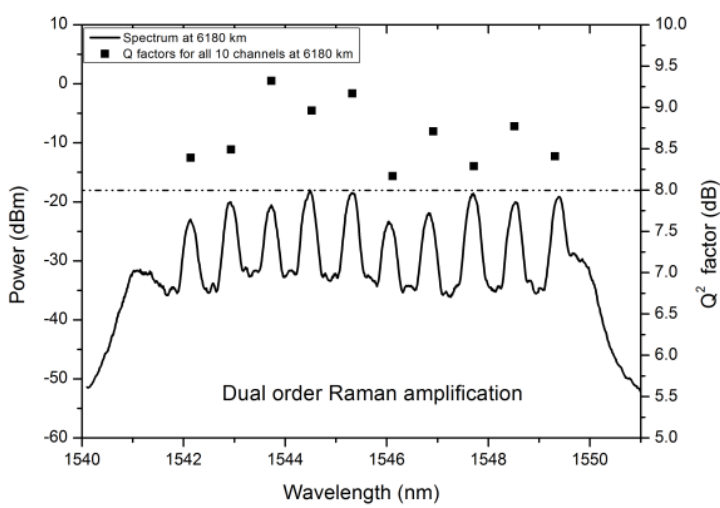

Figure 6. Spectrum of dual order Raman amplification system and $Q^{2}$ factors for all 10 channels at $6180 \mathrm{~km}$.

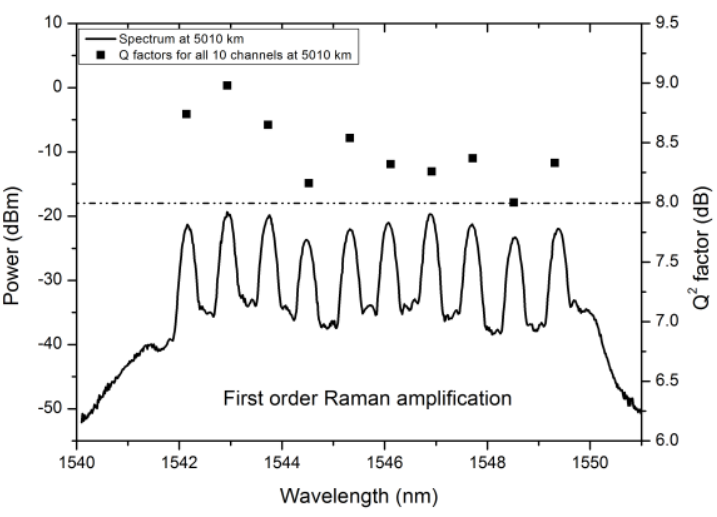

Figure 5. Spectrum of first order Raman amplification system and $Q^{2}$ factors for all 10 channels at $5010 \mathrm{~km}$.

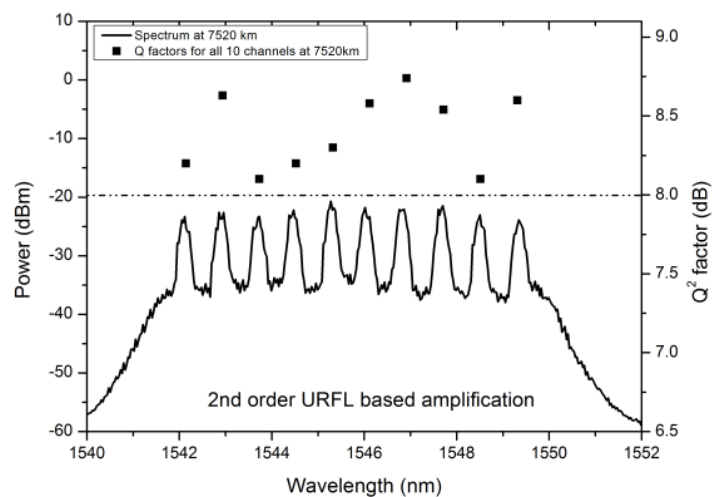

Figure 7. Spectrum of URFL based amplification system and $Q^{2}$ factors for all 10 channels at $7520 \mathrm{~km}$.

Figure 4 shows the transmission distances versus $\mathrm{Q}^{2}$ factors for the $193.5 \mathrm{THz}$ channel using the three different DRA techniques. In Figure 5, it shows an optical spectrum at the end of the loop and $\mathrm{Q}^{2}$ factors at $5010 \mathrm{~km}$ for all 10 channels using first order Raman amplification. This relatively poor performance compared to the other amplification techniques is essentially due to the relatively high $9.5 \mathrm{~dB}$ signal power variation which leads to a higher noise figure than the other techniques. Figure 6 shows equivalent results for dual order Raman amplification with an increased maximum reach of $6180 \mathrm{~km}$. The improved performance is due to much flatter signal power variation and improved noise figure compared with first order scheme. Figure 7 demonstrates the transmission performance at $7520 \mathrm{~km}$ using URFL based amplification. Please note that the noisy spectrum is due to the sweep time limit of the optical spectrum analyser gating mode used for these recirculating loop measurements. We believe this advantageous performance is because reduced signal power variation through the transmission span leads to a better OSNR. Meanwhile, there is no additional Q factor penalty caused by relative intensity noise (RIN) from the co-propagated component of fibre laser because the measured RIN of $1448 \mathrm{~nm}$ fibre laser is $-113 \mathrm{~dB} / \mathrm{Hz}$ and the total $1455 \mathrm{~nm}$ intra-cavity power is below $100 \mathrm{~mW}[12,16]$. The required total optical power for the first order, dual order and URFL based Raman amplification techniques are $600 \mathrm{~mW}, 1026$ $\mathrm{mW}$ and $1091 \mathrm{~mW}$ respectively. Although first order pumping technique requires the lowest optical power, the transmission performance is much worse than the other two. URFL based amplification requires similar total optical power to dual order Raman amplification but gives improved transmission performance. The application of URFL in amplification provides a flexible and simple approach by using a single wavelength pump [17].

\section{CONCLUSIONS}

We have experimentally compared ultra-long haul transmission of 10x118 Gbit/s DP-QPSK data on three different distributed Raman amplification techniques which use counter-propagated Raman pumping. For our $83 \mathrm{~km}$ transmission span, conventional first order gave a transmission distance of $5010 \mathrm{~km}$. System length was 
increased to $6180 \mathrm{~km}$ using dual order pumping and $7520 \mathrm{~km}$ using an ultra-long Raman laser configuration with counter propagating second order Raman pumping only. To the authors' knowledge, this is the first experimental comparison of these techniques. In addition to improved transmission performance the Raman fibre laser based amplification technique also has the advantage of requiring only a single pump wavelength. This scheme does however require additional components (FBGs) and required slightly higher total pump power than the conventional dual pump wavelength technique. We attribute the performance improvement with the ultra-long Raman fibre laser configuration to improved noise characteristics associated with the reduced signal power variations along the transmission span.

\section{ACKNOWLEDGEMENTS}

This work was funded by UK EPSRC Programme Grant UNLOC (Unlocking the capacity of optical communications) EP/J017582/1. The authors would like to thank Zhongyuan Sun and Prof. Lin Zhang for providing the FBGs used in the experiment.

\section{REFERENCES}

[1] E. Ip, et al.: Coherent detection in optical fiber systems, Opt. Express, vol. 16. pp. 753-791, Jan. 2008.

[2] J. C. Cartledge, et al.: Coherent detection in optical fiber systems, J. Lightwave Technol., vol. 30. pp. 3771-3778, Dec. 2012.

[3] Y. Hadjar, et al.: Noise figure tilt reduction in ultrawide WDM through second-order Raman amplification, IEEE Photon. Technol. Lett., vol. 16. pp. 1200-1202, April. 2004.

[4] P.M. Krummrich, R. E. Neuhauser, H. Bock, W. Fischler and C. Glingener: System performance improvements by codirectional Raman pumping of the transmission fiber, in Proc. ECOC 2001, Amsterdam, Netherlands, Sept 2001, paper TuA1.4.

[5] M.X. Ma, et al.: $240 \mathrm{~km}$ repeater spacing in a $5280 \mathrm{~km}$ WDM system experiment using $8 \times 2.5 \mathrm{~Gb} / \mathrm{s} \mathrm{NRZ}$ transmission, IEEE Photon. Technol. Lett., vol. 10. pp. 893-895, June. 1998.

[6] H. Bisserssur: Amplifier Technologies for Unrepeatered links, Submarine Transmission, in Proc. OFC 2013, Los Angeles, USA, March 2013, paper OTh4C.3.

[7] P. Rosa, P. Harper, N. Murray and J. Ania-Castanon: Unrepeatered $8 \times 40 \mathrm{~Gb} / \mathrm{s}$ transmission over 320km SMF-28 using ultra-long Raman fibre based amplification, in Proc. ECOC 2012, Amsterdam, Netherlands, Sept 2012, paper 4.04.

[8] I.D. Phillips, M. Tan, M.F.C. Stephens, M. E. McCarthy, E. Giacoumidis, S. Sygletos, P. Rosa, S. Fabbri, S. T. Le, T. Kanesan, S. K. Turitsyn, N. J. Doran, P. Harper and A. D. Ellis: Exceeding the NonlinearShannon Limit using Raman Laser Based Amplification and Optical Phase Conjugation, in Proc. OFC 2014, San Francisco, USA, March 2014, paper M3C1.

[9] L. Barker, A. E. El-Taher, M. Alcon-Camas, J. D. Ania-Castanon and P. Harper: Extended bandwidth for ultra-long haul Raman fibre lasers, in Proc. ECOC 2010, Torino, Italy, Sept 2010, paper, PP3.

[10] J. D. Ania-Castanon, et al.: Simultaneous spatial and spectral transparency in ultralong fiber lasers, Phys. Rev. Lett., vol. 101. pp.123903(1)-(4), Sept. 2008.

[11] T. J. Ellingham et al., Quasi-lossless Optical Links for Broad-band Transmission and data processing, IEEE Photon. Technol. Lett., vol. 18. pp. 268-270, Jan. 2006.

[12] V. Karalekas et al., Impact of nonlinear spectral broadening in ultra-long Raman fibre lasers, Opt. Express, vol. 15. pp. 16690-16695, Dec. 2007.

[13] J. -C. Bouteller, K. Bar, S. Radic, J. Bromage, Z. Wang and C. Headley: Dual-order Raman pump providing noise figure and large gain bandwidth, in Proc. OFC 2002, Anaheim, USA, March 2010, postdeadline paper FB3.

[14] J.-C. Bouteller et al.:Dual order Raman pump, IEEE Photon. Technol. Lett., vol. 15. pp. 212-214, Feb. 2003.

[15] M.F.C. Stephens, M. Tan, I.D. Phillips, S. Sygletos, P. Harper and N.J. Doran: 1THz-Bandwidth Polarization-Diverse Optical Phase Conjugation of 10x114Gb/s DP-QPSK WDM Signals, in Proc. OFC 2014, San Francisco, USA, March 2014, paper W3F6.

[16] B. Bristiel et al.:New Model of Noise Figure and RIN Transfer in Fiber Raman Amplifiers, IEEE Photon. Technol. Lett., vol. 18. pp. 980-982, April. 2006.

[17] M. Alcon-Camas, A.E.El-Taher, J.-D. Ania-Castanon and P. Harper: Gain bandwidth optimisation and enhancement in ultra-long Raman fibre laser based amplifiers, in Proc. ECOC 2010, Torino Italy, Sept 2010, paper, P1.17. 